\title{
Re-assessing the infection strategies of the entomopathogenic nematode Steinernema feltiae (Rhabditidae; Steinernematidae)
}

\author{
J. P. FAIRBAIRN*, A. FENTON, R. A. NORMAN and P. J. HUDSON \\ University of Stirling, Stirling, Scotland FK9 $4 L A$
}

(Received 18 October 1999; revised 7 Fanuary 2000 and 14 February 2000; accepted 14 February 2000)

\section{S U M M ARY}

Previous studies have indicated that between 60 and $80 \%$ of a population of entomopathogenic nematodes do not infect their insect hosts at any one period in time. Two hypotheses explain this behaviour: the first that there is a subpopulation of non-infectious nematodes and the second that the non-infectious group is created by inhibitory cues derived from infected insects. Through an experimental approach with the Galleria mellonella-Steinernema feltiae system we show that both mechanisms operate together. When conditions for infection were optimized, the sum of individual infection behaviours was similar to the number infecting as a population, implying observed infection rates are driven by intrinsic mechanisms. In addition, there was evidence that an infected host released a chemical cue into the environment which inhibited subsequent levels of infection. This degree of inhibition was independent of the number of infecting nematodes. Both these mechanisms are dynamic, so the observed proportion of infectious nematodes depended heavily on the time of exposure. The implications of these findings for both the design of laboratory trials and the use of entomopathogenic nematodes in biological control are discussed.

Key words: entomopathogenic nematodes, Steinernema feltiae, subpopulation, inhibitory cue, biological control.

\section{INTRODUCTION}

Entomopathogenic nematodes are used as biological control agents of insect soil pests such as sciarid mushroom flies (Lycoriella spp.) and the black vine weevil (Otiorhynchus sulcatus). Nematodes are typically applied in high numbers, for example 3 million infective juveniles per square metre are recommended to control the mushroom sciarid fly (MicroBio, UK). Although such a large number of nematodes are used to protect crops, laboratory studies have shown that the majority of the freeliving larvae are not infective (Bednarek \& Nowicki, 1986; Fan \& Hominick, $1991 a$; Bohan \& Hominick, $1995 a, b, 1996,1997$; Glazer, 1997). The aim of this study is to understand the infection process that causes this phenomenon.

Following the work of Bednarek \& Nowicki (1986) two hypotheses have arisen in the literature to describe the invasion strategies of these nematodes. The first states that infective juveniles can be divided into two subpopulations where one is infective and the other is not. To demonstrate this Bohan \& Hominick (1995a, b, 1996) showed that the overall proportion of the nematode Steinernema feltiae (Filipjev) infecting in a given time-period did not change and never exceeded $40 \%$, even when the

* Corresponding author: Institute of Biological Sciences, University of Stirling, Stirling, Scotland FK9 4LA. Tel: + 01786467832 . Fax: + 01786464994 .

E-mail: jpf2@stir.ac.uk initial number of nematodes applied was varied. Further support for a subpopulation comes from a series of experiments by Fan \& Hominick (1991 $a$ ), Mannion \& Jansson (1993) and Hay \& Fenlon (1995). However, these experiments did not take into account the mortality of nematodes during the time of the experiment, a factor which is known to be very high (Smits, 1996). By ignoring the mortality of nematodes over time it is impossible to evaluate the true proportion of infectious nematodes in the freeliving population as many of the nematodes will have died before they could infect.

The second hypothesis stems from work by Glazer (1997) and states that some part of the nematodeinfected host complex produces a chemical cue that results in infective juveniles in the surrounding environment not infecting hosts. He demonstrated that the number of infective juveniles infecting a fresh host, after exposure to an infected host, was reduced.

It should be noted that the experimental work carried out on the invasion tactics of entomopathogenic nematodes has largely focused on testing only one of these hypotheses (Fan \& Hominick, $1991 a$; Mannion \& Jansson, 1993; Bohan \& Hominick, $1995 a, b)$. For their subpopulation infection-model, Bohan \& Hominick (1995 $a, b)$ explicitly stated an assumption that mortality was unimportant over the short time-scale of infection and implicitly assumed that no host derived cues operated to reduce nematode infection. However, 
given that mortality may be significant (Smits, 1996) and the findings of Glazer (1997), we suggest that the conclusions of Bohan \& Hominick $(1995 a, b)$ and others. need re-evaluating.

These two hypotheses may not be mutually exclusive and, in this paper, we undertake a series of experiments to examine both. Specifically we designed experiments to answer the following questions. (1) Can a subpopulation be identified by investigating groups of invading nematodes? (2) Does the infection rate of individual nematodes vary temporally? (3) Do infected hosts release a chemical cue that prevents infection? (4) If so, is the rate of cue production dependent on the initial density of infecting nematodes?

By answering these questions our objective is to determine the major factors limiting the infection rate of entomopathogenic nematodes.

Entomopathogenic nematodes belonging to the families Steinernematidae and Heterorhabditidae are lethal obligate parasites of arthropods. Free-living infective stages known as infective juveniles carry symbiotic bacteria (Xenorhabdus sp.) in the anterior portion of the gut and can be attracted to the host by cues such as $\mathrm{CO}_{2}$, gut contents and cuticle (Lewis, Gaugler \& Harrison, 1992, 1993; Grewal, Gaugler \& Lewis, 1993). For S. feltiae, once contact has been made, the nematode infects the host via natural openings (mouth, spiracles or anus) and releases the symbiotic bacteria causing host death within $48 \mathrm{~h}$. The infective juveniles then feed on the soup of bacteria and host nutrients, develop into adults and reproduce, releasing around $2 \times 10^{4}$ infective juveniles into the environment 2 weeks following infection. Steinernematids are dioecious and require infection of at least one male and one female into the same host for reproduction to take place. However, Heterorhabditids are hermaphroditic and require only one individual to enter the host, although the subsequent generations reproduce sexually. In both cases, a single insect host represents both the feeding and breeding site for entomopathogenic nematodes.

\section{MATERIALS AND METHODS}

Steinernema feltiae were reared through larvae of the greater waxmoth Galleria mellonella according to methods described by Woodring \& Kaya (1988) and tested on late instar G. mellonella reared on a mixture of wheat middlings, honey, beeswax and yeast. $G$. mellonella lend themselves to experimentation with entomopathogenic nematodes because they are highly susceptible to infection (Bohan \& Hominick, 1996). Experiments have demonstrated a lack of immunity in this system (Fairbairn, unpublished data; Bohan, personal communication) but for other systems, such as the sciarid fly-S. feltiae system, there appears to be immunity to low levels of infection (Hay \& Fenlon, 1995).
All experiments were carried out at $20^{\circ} \mathrm{C}$, a temperature similar to that where $S$. feltiae is used in biological control.

\section{Experiment1: Can a subpopulation be identified by investigating groups of invading nematodes?}

Previous studies (Fan \& Hominick, $1991 a$; Bohan \& Hominick, $1995 a, b)$ reported two subpopulations for entomopathogenic nematodes following experiments involving large groups of infective juveniles. The simplest way of determining the presence of two subpopulations where one is infective and the other not is to perform replicated challenges of the host with one nematode. To validate the work of Bohan $\&$ Hominick $(1995 a, b)$ where they showed no change in the measured infectious proportion when groups of between 100 and 800 nematodes were challenged against Galleria, we undertook two extreme tests. The infectivity of single nematodes exposed to a single host was compared with that of 1000 nematodes exposed to a single host.

Nematodes were placed either singly or as a group of 1000 into $1.5 \mathrm{ml}$ Eppendorf tubes filled with moist sand. Singletons were collected under dissection microscope using a Hamilton $100 \mu \mathrm{m}$ syringe whilst groups of 1000 were obtained from suspensions of 4000 nematodes per $\mathrm{ml}$ with a $250 \mu \mathrm{l}$ pipette. Following the addition of these nematodes, the Eppendorf tubes were incubated at $20^{\circ} \mathrm{C}$ for $6 \mathrm{~h}$ to allow the nematodes time to disperse around the sand, reducing the activity levels of the nematodes that may have been enhanced during handling. A total of 388 replicates of single nematodes versus Galleria and 20 each of 1000 nematodes versus Galleria and control tubes were performed. A single Galleria was then placed into each tube and incubated for $24 \mathrm{~h}$ at $20^{\circ} \mathrm{C}$. We used such a small arena size to limit the effect of space in an attempt to ensure that the nematodes had the maximum opportunity to infect. A ventilation hole was provided in the lid of the tubes and they were placed into a plastic bag with moist cotton wool to maintain humidity. Host mortality was assessed after a further $48 \mathrm{~h}$ and the number of nematodes that became infective was determined by dissection of the dead Galleria. Nematode mortality was controlled for by placing populations of 1000 nematodes into tubes without hosts for $24 \mathrm{~h}$ and assessing the number of surviving nematodes under the microscope following sand washing (Bohan \& Hominick, 1995). The results were analysed using a $\chi^{2}$ test, to distinguish whether or not the same proportions of nematodes infected when in large numbers or as singletons.

\section{Experiment 2: Does the infection rate of individual} nematodes vary temporally?

Five hundred Eppendorf tubes were set up in the same manner as Exp. 1 with 1 nematode and 1 


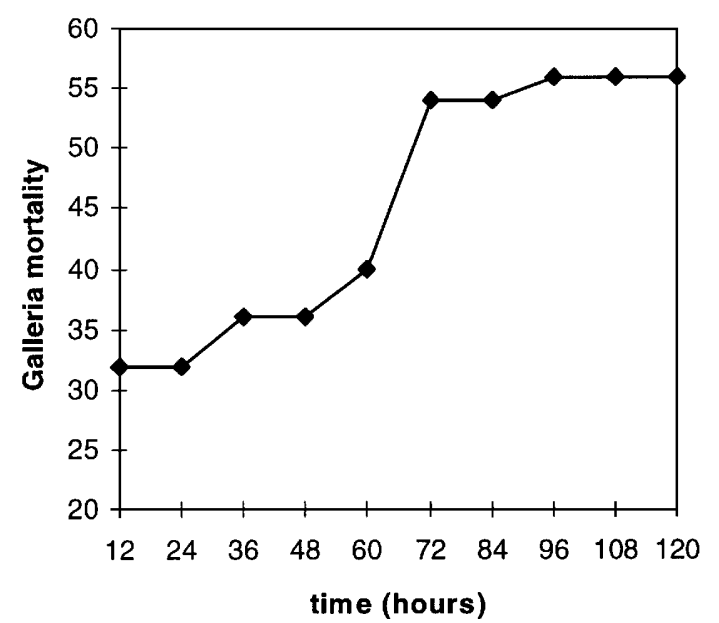

Fig. 1. The number of nematodes becoming infective over a 5 -day period. Galleria mortality as a percentage is plotted against time.

Galleria, incubated at $20^{\circ} \mathrm{C}$ for up to 5 days. Every $12 \mathrm{~h}, 50$ tubes were removed from the experiment, the Galleria extracted, washed and incubated for a further $48 \mathrm{~h}$ and mortality recorded. Controls for nematode mortality consisted of 50 tubes containing single nematodes but no host. At each $12 \mathrm{~h}$ interval, 10 tubes were washed to recover the nematodes and mortality determined under a dissection microscope. The number of new infections over the 5-day period was corrected for this mortality rate.

Experiment 3: Do infected Galleria release a chemical cue which prevents infection?

Galleria were injected with 250 nematodes in $10 \mu \mathrm{l}$ of sterile Ringers solution and placed on moist sand in similar arenas to those in Exps 1 and 2. Injecting Galleria has no effect on the subsequent infection rates of nematodes (Fairbairn, unpublished data). Injected Galleria were assigned to 1 of 2 treatments: incubated on the sand for 24 or $48 \mathrm{~h}$. Controls for each treatment consisted of Galleria injected with sterile Ringers with all treatments replicated 20 times. Following exposure on the sand, the infected Galleria were removed and 1000 nematodes pipetted onto the sand. These nematodes were left for $6 \mathrm{~h}$ to acclimatize after which a single fresh Galleria was added to the arena. These fresh Galleria were incubated in the tubes for $24 \mathrm{~h}$ and then removed, washed and further incubated for $48 \mathrm{~h}$. Infected Galleria were then dissected to establish the number of nematodes that had infected. The results were analysed using an ANOVA test where each treatment was tested against its corresponding control.

Experiment 4: Is the production of a cue dependent upon the number of invading nematodes?

Galleria were injected with 10, 50, 100 and 250 nematodes and placed into sand-filled Eppendorf tubes in a similar manner to that described above.
The tubes were incubated at $20^{\circ} \mathrm{C}$ for $48 \mathrm{~h}$ after which time the Galleria were removed and 1000 nematodes placed onto the sand for $6 \mathrm{~h}$, as in Exp. 3 . Fresh Galleria hosts were then added and incubated at $20^{\circ} \mathrm{C}$ for a further $24 \mathrm{~h}$. Galleria were then removed, washed and dissected after $48 \mathrm{~h}$ to determine the number of nematodes infecting. Again, each condition was replicated 20 times. The results were analysed by GLM.

\section{RESULTS}

Experiment 1: Can a subpopulation be identified by investigating groups of invading nematodes?

There was no significant difference $(P=0 \cdot 44$, D.F. $=1)$ in the number of nematodes infecting as singletons $(17.5 \%$ ) or as a group (mean $14.3 \%$ S.D. $=8 \cdot 3)$. This demonstrated that the infective behaviour of nematodes is independent of factors arising from the presence of other nematodes.

\section{Experiment 2 : Does the infection rate of individual nematodes vary temporally?}

After adjusting for nematode mortality, which reached $50 \%$ during the experiment, $32 \%$ of the infective juveniles infected within the first $12 \mathrm{~h}$. After this initial infection, the rate at which nematodes infected the hosts increased in a sigmoidal manner (logistic regression $P<0 \cdot 001$ ) over a period of 4 days until, at the end of the experiment, $56 \%$ of the population had infected (Fig. 1). Following the initial infection, the subsequent time to infect followed a continuous distribution with a mean of $64.9 \mathrm{~h}$ (s.D. $=16 \cdot 2, n=10$ ) showing that the proportion of non-infectious nematodes is not static.

Experiment 3 : Do infected Galleria release a chemical cue which prevents infection?

Data were normalized with square-root transformation. There was no significant difference in the number of nematodes infecting following exposure for $24 \mathrm{~h}$ compared to controls $\left(\mathrm{F}_{1,38}=0 \cdot 27\right.$, $P=0 \cdot 608$; Fig. 2A) but there was a significant reduction in the number infecting after $48 \mathrm{~h}$ presentation $\left(\mathrm{F}_{1,38}=4.41, P=0.042\right.$; Fig. 2B). From this, we conclude that infected Galleria emitted a cue which remained on the sand and inhibited the infection behaviour of nematodes following the first $48 \mathrm{~h}$, but not the first $24 \mathrm{~h}$, post-infection.

\section{Experiment 4: Is the production of a cue dependent upon the number of invading nematodes?}

Once again, data were normalized through squareroot transformation. The number of nematodes infecting a fresh Galleria following the presentation of infected Galleria was not significantly different between treatment groups $\left(\mathrm{F}_{3,76}=0 \cdot 18, P=0 \cdot 91\right.$; 


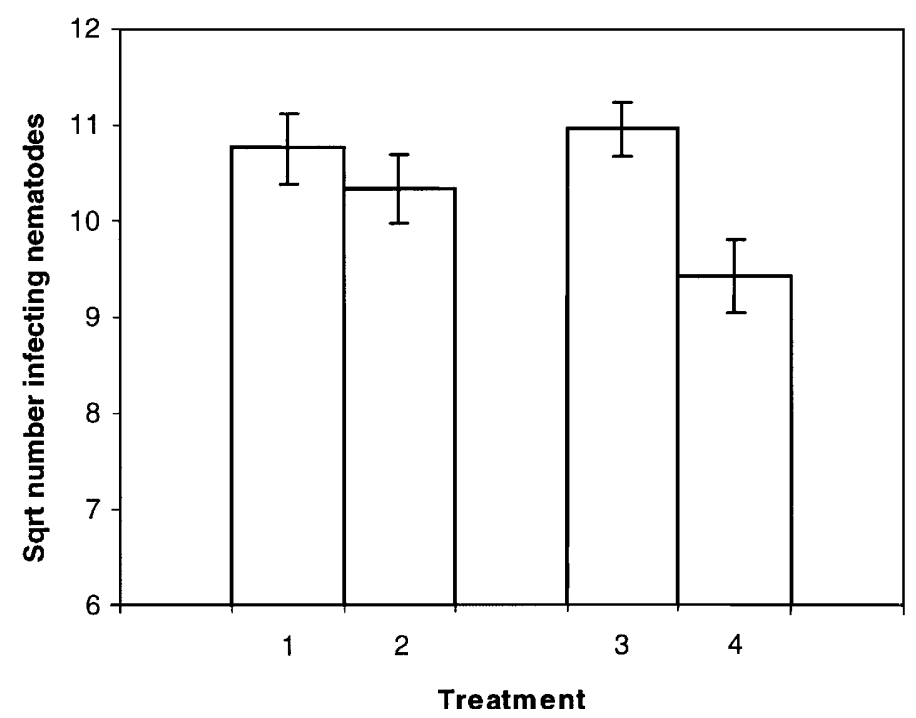

Fig. 2. Square root mean number of nematodes infecting Galleria which had been placed on sand previously exposed to a non-infected Galleria (treatment 1) and an infected Galleria (treatment 2) for 24 h and a non-infected Galleria (treatment 3) and an infected Galleria (treatment 4) for $48 \mathrm{~h}$ with standard errors shown.

Fig. 3) where square root mean number of nematodes infecting fresh Galleria following injection with 10, 50,100 and 250 nematodes was $5 \cdot 77$ (s.E. $\pm 0 \cdot 41$ ), $5 \cdot 61$ $($ s.E. $\pm 0 \cdot 38), 5 \cdot 27($ s.E. $\pm 0 \cdot 42)$ and $5 \cdot 16$ (s.E. $\pm 0 \cdot 38)$ respectively. Therefore the number of infecting nematodes was found not to be dependent on the number of nematodes which had infected the primary hosts.

\section{DISCUSSION}

The objective of this work was to determine the major factors limiting infection rates of entomopathogenic nematodes. The two hypotheses examined were: (1) an intrinsic mechanism, acting within each individual infective juvenile results in a proportion being infective at any one time, (2) an extrinsic chemical cue, arising from an infected host, prevents infective juveniles becoming infective.

Experiments demonstrated that the infective juveniles of $S$. feltiae exhibit considerable variation in the time until they become infective. The initial dynamics of the system were not observed as nematodes used in experiments were from a population that was up to 6 days old. However, after this time an initial group of the infective juvenile population was seen to be immediately infectious with the remainder subsequently joining the infectious class, following a continuous distribution with time. This behaviour was shown not to be the result of interactions between individual nematodes.

There are 4 possible explanations for these findings. Firstly, the arena size may have been too big to allow more than the observed percentage of nematodes to encounter the host within the timescale of the experiment. This is unlikely, since in a similar experiment Bohan \& Hominick (1996) found that hosts and nematodes occupied the same space when placed into $30 \mathrm{ml}$ Universal tubes and the $2 \mathrm{ml}$ Eppendorf tubes used in this study were considerably smaller than those used by Bohan. Also, nematodes have the potential to move up to $16 \mathrm{~cm}$ through sand within $24 \mathrm{~h}$ (Koch-Osborne, unpublished data). The second explanation for the observed proportion infecting may be that hosts exhibit differential susceptibility to infection from nematodes. However, Galleria were chosen for this experiment specifically because they have been demonstrated to lack immunity to $S$. feltiae (Bohan, personal communication) although this may not be true for all systems and nematode species (Hay \& Fenlon, 1995; Campbell et al. 1999). The third possibility is that hosts may be differentially attractive to nematodes, but this would create a non-random distribution of nematodes between hosts as would have been the case for immunity. Bohan \& Hominick (1995a,b) explicitly used a Poisson model to describe the distribution of nematodes, thereby demonstrating that nematodes were distributed randomly amongst hosts. Finally, the infective behaviour of nematodes may be dependent on intrinsic mechanisms which act within each individual nematode. Our findings best support this latter hypothesis and we suggest that it is this intrinsic mechanism that drives the infection process of the entomopathogenic nematode S. feltiae.

Further experiments (3 and 4) showed that this infectious behaviour of infective juveniles could be altered by extrinsic factors. Previous studies have shown that infective juveniles react positively to cues derived from a host in a fashion likely to enhance infection (Lewis, Gaugler \& Harrison, 1992, 1993; Grewal, Gaugler \& Lewis, 1993). Here we have shown that infective juveniles can react negatively to cues derived from the infected host-nematode complex to decrease the likelihood of infection. We 
showed a reduction in the number of nematodes infecting a fresh (uninfected) host following exposure to an infected host after $48 \mathrm{~h}$. This finding is in agreement with the results of Glazer (1997) who first proposed that a chemical cue was produced from an infected cadaver inhibiting subsequent infectivity of nematodes. We can be certain that the cue was of a chemical nature and not a physical one, by the fact that the infected host had been removed from the arena, leaving no physical traces and the subsequent group of nematodes was still inhibited from infecting. The degree of inhibition of infective behaviour was independent of the number of nematodes which infected the initial host. Hence, the mechanism of cue production does not depend on the density of invading nematodes but may either depend on bacteria density or else be produced in an all-or-nothing manner, requiring only that the host be infected.

This work is the first to show that both of the proposed mechanisms explaining the proportion of nematodes found to infect are operating. With this in mind the work of Fan \& Hominick (1991a,b), Mannion \& Jansson (1993) and Bohan \& Hominick $(1995,1996)$ where they never found more than $40 \%$ of nematodes infecting may require re-evaluation, since they do not correctly explain the observed proportions of infecting nematodes. A cue may have been acting to reduce the number of nematodes infecting and, as the dynamic nature of the infectious proportions was not taken into account, the estimates for the number of nematodes which were infectious in each group will also have been underestimated.

The implications of these findings for biological control are promising. Firstly, we have shown that the proportion of nematodes that are infectious is not fixed but dynamic. Therefore nematodes which do not infect immediately following application will become infective over time. In mushroom houses populations have been shown to survive for periods extending 1 week with low rates of decline (R. L. Gwynn, unpublished data) so, in these cases the total number of nematodes which will become infective will be upwards of $50 \%$.

Secondly, the existence of an inhibitory cue may result in control being inversely dependent on host density. When a large density of hosts exists (exactly the situation when control should be at its maximum) the production of an inhibitory cue will reduce the number of nematodes available for control. However, this may have a minor effect on their use in biological control for two reasons. Firstly, over a period of $48 \mathrm{~h}$ the reduction in the infective behaviour was very small and many nematodes were still able to infect. Secondly, entomopathogenic nematodes are highly virulent and host death can be caused by very few invading nematodes so even when a majority of nematodes is inhibited a few may still be active enough to kill hosts.
Finally, entomopathogenic nematodes appear to exhibit mixed strategies of host infection. It remains to be shown whether these strategies affect the efficiency of these nematodes as biological control agents and a combination of modelling and experiments designed to incorporate both of these behaviours need to be adopted to investigate this.

We would like to thank D. Bohan and R. Gwynn (MicroBio,UK) for their technical advice. This work is supported by a grant from NERC.

\section{REFERENCES}

BEDNAREK, A. \& NOWICKI, T. (1986). Effect of intrapopulation factors in the nematodes Steinernema feltiae (Steinernematidae) on intensity of infection. Zeszyty Problemowe Postepow Nauk Rolniczych 323, 199-210.

BOHAN, D. A. \& HOMINiCK, W. M. (1995a). Intrapopulation infectious structure and temporal variation in Steinernema feltiae. COST 819, Publication of the European Communities, Luxembourg.

Bohan, D. A. \& Hominick, W. M. (1995b). Examination of an infectious proportion amongst populations of Steinernema feltiae (site 76 strain) infective stages. Parasitology 111, 617-625.

BOHAN, D. A. \& HOMINICK, W. м. (1996). Investigations on the presence of an infectious proportion amongst populations of Steinernema feltiae (site 76 strain) infective stages. Parasitology 112, 113-118.

BOHAN, D. A. \& HOMINick, W. M. (1997). Long-term dynamics of infectiousness within the infective-stage pool of the entomopathogenic nematode Steinernema feltiae (site 76 strain) Filipjev. Parasitology 114, 301-308.

CAMPBELL, J. F., KOPPENHOFER, A. M., KAYA, H. K. \& CHINNASRI, B. (1999). Are there temporarily noninfectious dauer stages in entomopathogenic nematode populations: a test of the phased infectivity hypothesis. Parasitology 118, 499-508.

FAN, x. \& HOMINICK, W. M. (1991 $a$ ). Efficiency of the Galleria (wax moth) baiting technique for recovering infective stages of entomopathogenic rhabditids (Steinernematidae and Heterorhabditidae) from sand and soil. Revue de Nematologie 14, 381-387.

FAN, x. \& HOMINick, W. M. (1991 b). Effects of low storage temperature on survival and infectivity of two Steinernema species (Nematoda: Steinernematidae). Revue de Nematologie 14, 407-412.

GLAZER, I. (1997). Effects of infected insects on secondary invasion of steinernematid entomopathogenic nematodes. Parasitology 114, 597-604.

GRewal, P. S., GAugler, R. \& Lewis, E. E. (1993). Host recognition behavior by entomopathogenic nematodes during contact with insect gut contents. Fournal of Parasitology 79, 495-503.

HAY, D. B. \& FENLON, J. s. (1995). A modified binomial model that describes the infection dynamics of the entomopathogenic nematode Steinernema feltiae (Steinernematidae; Nematoda). Parasitology 111, 627-633. 
LEWIS, E. E., GAUGLER, R. \& HARRISON, R. (1992).

Entomopathogenic nematode host finding: response to host contact cues by cruise and ambush foragers.

Parasitology 105, 309-315.

LEWIS, E. E., GAUGLER, R. \& HARRISON, R. (1993).

Response of cruiser and ambusher entomopathogenic nematodes (Steinernematidae) to host volatile cues.

Canadian Fournal of Zoology-Revue Canadienne De

Zoologie 71, 765-769.

MANNION, C. M. \& JANSSON, R. K. (1993). Within-root mortality of Cylas formicarius (Coleoptera, Apionidae) by entomopathogenic nematodes. Fournal of Economic Entomology 86, 722-729.

SMits, P. H. (1996). Post-application persistence of entomopathogenic nematodes. Biocontrol Science and Technology 6, 379-387.

Woodring, J. L. \& KaYa, H. K. (1988). Steinernematid and Heterorhabditid Nematodes: A handbook of Techniques. Southern Cooperative Series Bulletin 331. Fayetteville, Arkansas Agricultural Experimental Station, AR. 